\title{
TEORIA DOS JOGOS: IDENTIFICAÇÃO DO PONTO DE EQUILÍBRIO DE NASH EM JOGOS BIMATRICIAIS DE INDICADORES ECONÔMICOS E SOCIAIS
}

\author{
FERNANDA KREUZBERG \\ Doutoranda pelo Programa de Pós-Graduação em Controladoria e Contabilidade \\ Universidade de São Paulo \\ E-mail: fernandakreuzberg@gmail.com \\ MOACIR MANOEL RODRIGUES JUNIOR \\ Professor do Programa de Pós-Graduação de Métodos Numéricos em Engenharia \\ Universidade Federal do Paraná \\ E-mail: moacir_ro@hotmail.com \\ NELSON HEIN \\ Professor do Programa de Pós Graduação em Ciências Contábeis \\ Universidade Regional de Blumenau \\ E-mail: hein@furb.br \\ VOLMIR EUGÊNIO WILHELM \\ Professor do Programa de Pós-Graduação de Métodos Numéricos em Engenharia \\ Universidade Federal do Paraná \\ E-mail: volmir@mat.ufpr.br
}

\begin{abstract}
RESUMO
Atualmente as empresas precisam pensar dentro de seu sistema de gestão em satisfazer não apenas os interesses econômicos da mesma, mas satisfazer os interesses sociais, vislumbrando a sustentabilidade e perenidade das atividades. Nesta discussão a presente pesquisa apresenta por objetivo identificar o Ponto de Equilíbrio de Nash no jogo bimatricial de indicadores econômicos e indicadores sociais das empresas do setor de Utilidade Pública listadas na BM\&FBovespa. Realizou-se a pesquisa com uma amostra de 26 empresas. A escolha dos indicadores para a avaliação de desempenho econômico está pautada em indicadores de rentabilidade e de mercado e para a avaliação de desempenho social, utilizou-se os indicadores oriundos da DVA. Para estimar os ganhos dos indicadores se utilizou os parâmetros da regressão linear múltipla. Os resultados obtidos destacaram a existência de pelo menos um ponto de equilíbrio para os anos analisados, e em especial os modelos propuseram dois pontos de equilíbrio para 2009 e 2011, e três pontos para 2010. Destaca-se que o modelo proposto confirmou a hipótese da existência de pelo menos um ponto de equilíbrio, e assim a pesquisa consegue satisfazer seu objetivo de que é possível atender tanto aos aspectos econômicos quanto aos aspectos sociais das empresas quando se toma como informações básicas os indicadores.
\end{abstract}

Palavras-Chave: Jogos Bimatriciais. Desempenho Econômico. Desempenho Social.

\section{ABSTRACT}

Today, companies need to think within your management system to meet not only the economic interests of the same, but satisfy the social interests of the sustainability and longevity of the activities. This discussion presents the current research aims to identify the point of Nash equilibrium in game bi-matrix economic indicators and social indicators of companies in the Utilities sector listed on the BM\&FBovespa. Was conducted the research with a sample of 26 companies. The choice of indicators for the assessment of economic performance is guided by indicators of profitability and market share and to assess social performance, we used the indicators derived from the SVA. Estimate the gains among the 
indicators we used the parameters of multiple linear regression. The results highlighted the existence of at least one equilibrium point for the years analyzed, and in particular the models proposed two equilibrium points for 2009 and 2011, and three points for 2010. It is noteworthy that the proposed model confirmed the hypothesis of the existence of at least one equilibrium point, and so the search can meet your goal that you can meet both the economic aspects and social aspects of business when it takes as the basic information indicators.

Key-words: Games Bi-Matrix. Economic Performance. Social Performance

\section{INTRODUÇÃO}

Durante a última década houve um aumento na pressão exercida sobre as empresas, de maneira que ampliem o seu foco na sustentabilidade e responsabilidade social dos negócios, para além do desempenho financeiro (Lee \& Saen, 2012). O conceito de desenvolvimento sustentável se tornou popular na década de 80, devido à definição apresentada em 1987, pela World Commission on Environment and Development - WCED (Comissão Mundial sobre Meio Ambiente e Desenvolvimento) das Nações Unidas, também conhecido como o Relatório de Brundtland. A definição apresentada envolve um conceito ético em relação ao combate à pobreza e proteção ao meio ambiente, compreendendo o nível macro (Baumgartner \& Ebner, 2010; Clarke-Sather, Hutchins, Zhang, Gershenson, \& Sutherland, 2011).

Conforme Claro, Claro e Amâncio (2008), após a definição apresentada pela Comissão Brundtland, surgiram diversas outras definições, sendo que muitas outras existirão no futuro, porém, o ponto em comum está na análise das dimensões que compõem a sustentabilidade.

Ebner e Baumgartner (2006) afirmam que no decorrer das últimas décadas a sustentabilidade tornou-se um termo muito popular na economia moderna. Lee e Saen (2012) corroboram que a adoção de práticas de sustentabilidade garante uma vantagem competitiva bem como a promulgação da eficiência das empresas em um cenário turbulento de negócios. Da mesma forma, Ioannou e Serafeim (2012) salientam que a adoção de práticas sustentáveis pelas empresas, além de ocasionar uma vantagem competitiva e incrementar o desempenho financeiro de longo prazo, pode ocasionar uma melhora no valor econômico gerado pelas empresas.

A ciência vem reforçando o foco da sustentabilidade empresarial, principalmente no que tange à diferenciação dos termos de sustentabilidade (especificamente a dimensão social) e responsabilidade social. Ebner e Baumgartner (2006) efetuaram um levantamento bibliográfico, e apontaram como sendo comum a discussão dessa diferenciação dos termos, e que na visão de diversos autores, ambos os conceitos apresentam abordagens distintas. Nesse sentido, Baumgartner e Ebner (2010) corroboram que a abordagem do desenvolvimento sustentável incorporado por uma organização é denominado de sustentabilidade empresarial, possuindo três pilares: econômico, ambiental e social.

A sustentabilidade corporativa é definida a partir do atendimento das necessidades atuais, diretas e indiretas dos stakeholders (acionistas, colaboradores, clientes, sociedades) bem como do atendimento das necessidades dos futuros stakeholders da empresa (Dyllick \& Hockerts, 2002). Portanto, para atender a este objetivo as empresas necessitam manter o seu crescimento econômico, social e ambiental. Nesse sentido, surge o conceito do Triple-Bottom Line, em que prevalece a perspectiva de integração dos aspectos econômicos, sociais e ambientais.

De acordo com Krajnc e Glavic (2005) as empresas utilizavam para um acompanhamento dos resultados de suas atividades apenas indicadores financeiros. Porém, para atender às exigências de seus agentes (fornecedores, empregados, bancos, seguradoras, acionistas, sociedade em geral), as empresas começaram a adotar práticas mais sustentáveis. Conforme Callado (2010) e Callado e Fensterseifer (2010), a motivação para a adoção de práticas sustentáveis consiste na continuidade das empresas que não é mais garantida apenas 
pela lucratividade. Influenciada por ações de sustentabilidade, a empresa pode estender as suas atividades por um período maior, gerando um aumento no valor de mercado das ações.

O estudo de Lo (2010) buscou analisar a eficiência das empresas norte americanas sustentáveis em comparação com as empresas não sustentáveis, pela avaliação da rentabilidade, utilizando o modelo de Análise Envoltória de Dados (DEA). A autora concluiu que as empresas sustentáveis apresentaram um melhor desempenho nos modelos de rentabilidade. $\mathrm{O}$ estudo compreende sustentabilidade ambiental a existência deste equilíbrio entre as ações de cunho econômico para com as ações de cunho social e ambiental.

Diante das diversas abordagens direcionadas para o conceito de sustentabilidade empresarial, o presente estudo adota o tradicional tripé da sustentabilidade que é composto pelas dimensões econômica, social e ambiental. A adoção desse tripé se justifica por ser esta a classificação mais consolidada na literatura até o momento. Efetuou-se a exclusão da dimensão ambiental no contexto da sustentabilidade empresarial, pelo fato de a sustentabilidade econômica ser o primeiro critério de análise (devido ao seu foco pautado na maximização dos resultados da empresa). Além disso, a dimensão social representa o mais alto critério de responsabilidade social, de maneira a contribuir com o bem estar da sociedade incluindo o conjunto de stakehoders (Aupperle; Carroll \& Hatfield, 1985; Carroll, 1991).

Diante do exposto apresenta-se a seguinte questão problema: Qual o Ponto de Equilíbrio de Nash no jogo bimatricial de indicadores econômicos e indicadores sociais das empresas do setor de Utilidade Pública listadas na BM\&FBovespa? No intuito de responder esta problemática a presente pesquisa apresenta por objetivo identificar o Ponto de Equilíbrio de Nash no jogo bimatricial de indicadores econômicos e indicadores sociais das empresas do setor de Utilidade Pública listadas na BM\&FBovespa.

Conforme Bontempo (1997) a teoria dos jogos é um instrumento matemático, mas que vem sendo estudado de maneira intensa também nas aplicações de teoria econômica, bem como em outras áreas do conhecimento, como por exemplo, a contabilidade. No que tange sua aplicação destaca-se o estudo de Brandt, Fischer, Harrenstein e Shoham (2009), que utilizouse a teoria dos jogos para determinar um Ponto de Equilíbrio de Nash entre o desempenho econômico e o desempenho social das empresas.

A análise do ponto de equilíbrio verifica as estratégias que as empresas deveriam assumir para alcançar os ganhos máximos com a minimização de suas perdas, tanto no desempenho econômico quanto no desempenho social de maneira simultânea. A delimitação de um ponto de equilíbrio permite as empresas atingir em um dado momento tanto ganhos máximos no desempenho econômico quanto no desempenho social, de modo a não ocasionar perdas a nenhum dos jogadores (Nash, 1950, 1951; Simonsen, 1994; Fiani, 2004; Lins \& Calôba, 2006).

No âmbito da análise do desempenho social, verifica-se ainda um grande número de estudos inconclusivos acerca dessa relação (Arlow \& Gannon, 1982; O’Bannon, 1997). Foram efetuadas importantes descobertas por Aupperle et al. (1985), Ullmann (1985) e Arlow e Gannon (1992), porém a discussão da relação do desempenho econômico-financeiro e desempenho social ainda não está completamente consolidada. Verifica-se uma diminuição nos estudos que apontam uma relação negativa entre o desempenho econômico e social. Com os resultados apontados por Ullmann (1985), Griffin e Mahon (1997), Roman, Hayibor e Agle (1999) confirma-se a afirmação anteriormente emitida por Cochran e Wood (1984). Cochran e Wood (1984) afirmam que os pesquisadores não chegaram a um consenso sobre a relação entre essas variáveis, ou ainda não existe uma relação clara entre o desempenho econômico e social, sendo esta uma importante discussão para a gestão empresarial.

\section{TEORIA DOS JOGOS}

O princípio da teoria dos jogos, considerando sua desvinculação direta com a Teoria das Probabilidades, ocorreu em meados do século XX, pelo matemático John Von Neumann, mediante a introdução da perspectiva de resolução de conflitos de interesse através da 
matemática (Souza, 2003). Porém ela tomou impulso a partir de 1944, quando Von Neumann e Oskar Morgenstern o livro "Theory of Games and Economic Behavior".

Conforme Fiani (2004) a teoria dos jogos consiste em um método aplicado ao processo de tomada de decisão quando dois ou mais agentes que interagem entre si (Fiani, 2004). Existe ai as diferentes abordagens dentro da Teoria dos Jogos, considerando a forma de interação entre jogadores, Jogos Não-Cooperativos e Jogos Cooperativos. Para a resolução de um jogo Não- Cooperativo que presume existe disputa entre os Jogadores não possibilitando acordos entre eles, faz-se necessário, primeiramente, de uma matriz com os ganhos e perdas dos jogadores. Assim, o Jogador "A" escolhe suas estratégias conforme dispostas nas linhas $(m)$, enquanto que o Jogador "B" efetua suas escolhas baseada nos ganhos apresentados nas colunas (n) (Lins \& Calôba, 2006).

Os jogos de soma zero consideram que a matriz de ganhos e perdas do Jogador A é oposta a matriz de ganhos e perdas do Jogador B. Entretanto, costumeiramente os ganhos e perdas entre os jogadores não são simétricos. Desta forma, percebendo que o modelo utilizado gerará matrizes não opostas, ou seja, o modelo utilizado é de soma diferente de zero, será necessária a avaliação dos resultados por meio de jogos bimatriciais recaindo na análise do ponto de equilíbrio de Nash.

Na matriz de jogo para o modelo de Equilíbrio de Nash, os Jogadores são identificados como indicadores sociais (Jogador A) e indicadores econômicos (Jogador B). Nesse caso, considera-se uma matriz de pares ordenados refletidos em ganhos de $\mathrm{A}\left(a_{i j}\right)$ e ganhos de $\mathrm{B}\left(b_{j i}\right)$, conforme apresentado na matriz da Tabela 1 .

Tabela 1 - Matriz do jogo

\begin{tabular}{|c|c|c|c|c|c|}
\hline & \multicolumn{4}{|c|}{ Jogador B (Indicadores Econômicos) } \\
\hline & & Indicador 1 & Indicador 2 & $\cdots$ & Indicador $n$ \\
\hline \multirow{4}{*}{$\begin{array}{c}\text { Jogador A } \\
\text { (Indicadores } \\
\text { Sociais) }\end{array}$} & Indicador 1 & $\left(a_{11} ; b_{11}\right)$ & $\left(a_{12} ; b_{21}\right)$ & $\ldots$ & $\left(a_{n} ; b_{n}\right)$ \\
\hline & Indicador 2 & $\left(a_{21} ; b_{12}\right)$ & $\left(a_{22} ; b_{22}\right)$ & $\cdots$ & $\left(a_{n} ; b_{n}\right)$ \\
\hline & $\vdots$ & $\vdots$ & $\vdots$ & $\because$ & $\vdots$ \\
\hline & Indicador $m$ & $\left(a_{m} ; b_{m}\right)$ & $\left(a_{m} ; b_{m}\right)$ & $\cdots$ & $\left(a_{m n} ; b_{m n}\right)$ \\
\hline
\end{tabular}

Fonte: Elaboração própria.

A solução do jogo consiste em identificar o par ordenado cujo ganho seja máximo entre as alternativas e a perda seja mínima entre as estratégias do oponente (Simonsen, 1994). Os valores para identificar o Ponto de Equilíbrio foram determinados por meio de Regressão Linear Múltipla. A partir, deste método, utilizam-se os valores dos Coeficientes Beta Padronizados (Beta Standarized).

A utilização dos valores dos Coeficientes Beta Padronizados é abordada por Maroco (2003: 391) ao inferir que "[...] a simples comparação dos coeficientes de regressão para avaliar a importância de cada variável independente no modelo não é válida". Justifica-se, a utilização desses valores dos Coeficientes Beta Pradonizados, pois as variáveis dependentes apresentam diferentes magnitudes. Dessa forma, "para que a importância de cada variável no modelo possa ser comparada, é necessário usar as variáveis standardizadas (ex. $Z_{i j}=$ $\left.\left(X_{i j}-\bar{X}_{l}\right) / S_{X_{i}}^{\prime}\right)$ no ajustamento do modelo ou então standardizar os coeficientes de regressão" (Maroco, 2003: 391).

Desse modo, para os fins deste estudo se considera apenas os valores de Beta Standard, sem considerar a eficácia do modelo e a significância das variáveis, pois objetiva-se apenas averiguar qual é a relação existente entre os indicadores e não os níveis de significância.

Nesse sentido o modelo construído se apresenta no formato descrito abaixo. 
Sendo $\mathrm{X}_{\mathrm{i}}$ variável dependente representando os $i$-ésimos indicadores sociais, $\mathrm{Y}_{\mathrm{i}}$ variável independente representativa dos $j$-ésimos indicadores econômicos temos a formação da seguinte equação:

$$
\mathrm{X}_{\mathrm{i}}=a_{0 \mathrm{i}}+a_{1 \mathrm{i}} Y_{1}+a_{2 \mathrm{i}} Y_{2}+\cdots+a_{\mathrm{ni}} Y_{n}+\varepsilon_{i}
$$

Com $i=1, \cdots, m$. Assim são estabelecidos os Coeficientes Normalizados da equação $\alpha_{1 \mathrm{i}}, \alpha_{\mathrm{i} 2}, \cdots, \alpha_{\mathrm{ni}}$, da seguinte forma:

$$
\alpha_{\mathrm{ij}}=a_{\mathrm{ij}}\left(\frac{s_{Y_{j}}}{s_{X_{i}}}\right)
$$

Estes coeficientes $\alpha_{i j}$ representam os ganhos dos indicadores sociais $X_{i}$ (estratégias) por parte do Jogador A, tendo em vista as $Y_{j}$ estratégias do jogador B.

De forma semelhante, para os indicadores econômicos, foram consideradas as variáveis $Y_{j}$ como as variáveis dependentes do modelo que representa os $j$-ésimos indicadores econômicos, bem como $X_{i}$ a variável independente representando os $i$-ésimos indicadores sociais das empresas analisadas. Tem-se assim a equação:

$$
\mathrm{Y}_{\mathrm{j}}=b_{0 \mathrm{j}}+b_{1 \mathrm{j}} X_{1}+b_{2 \mathrm{j}} X_{2}+\cdots+b_{\mathrm{mj}} X_{m}+\varepsilon_{j}
$$

Com $j=1, \cdots, n$. É possível obter os Coeficientes Normalizados da equação $\beta_{1 \mathrm{j}}, \beta_{2 \mathrm{j}}, \cdots, \beta_{\mathrm{nj}}$, calculados como:

$$
\beta_{\mathrm{ij}}=b_{\mathrm{ij}}\left(\frac{s_{X_{i}}}{s_{Y_{j}}}\right)
$$

De forma análoga ao que foi considerado para as estratégias de $X_{i}$ temos que as regressões identificam o valor de pagamento para $Y_{j}$ caso o jogador B o escolha como estratégia (Indicadores Econômicos), para todas as opções (Indicadores Sociais) do Jogador A.

A Matriz de comparação, foi adaptada da Tabela 1, e é construída conforme a Tabela 2.

Tabela 2 - Matriz de Comparação

\begin{tabular}{c|c|cccc}
\hline \multicolumn{2}{c|}{} & \multicolumn{4}{c}{ Jogador B (Indicadores Econômicos) } \\
\cline { 3 - 6 } \multicolumn{2}{c|}{} & $Y_{1}$ & $Y_{2}$ & $\ldots$ & $Y_{\mathrm{n}}$ \\
\hline \multirow{3}{*}{ Jogador A } & $X_{1}$ & $\left(\alpha_{11} ; \beta_{11}\right)$ & $\left(\alpha_{12} ; \beta_{21}\right)$ & $\ldots$ & $\left(\alpha_{n} ; \beta_{n}\right)$ \\
(Indicadores & $X_{2}$ & $\left(\alpha_{21} ; \beta_{12}\right)$ & $\left(\alpha_{22} ; \beta_{22}\right)$ & $\ldots$ & $\left(\alpha_{n} ; \beta_{n}\right)$ \\
Sociais) & $\vdots$ & $\vdots$ & $\vdots$ & $\ddots$ & $\vdots$ \\
& $X_{\mathrm{m}}$ & $\left(\alpha_{m} ; \beta_{m}\right)$ & $\left(\alpha_{m} ; \beta_{m}\right)$ & $\ldots$ & $\left(\alpha_{m n} ; \beta_{m n}\right)$ \\
\hline
\end{tabular}

Fonte: Elaboração própria.

Para determinar o Ponto de Equilíbrio de Nash em um jogo bimatricial, Simonsen (1994:414) salienta que se deve identificar "[...] uma casa onde o ganho de A seja máximo na coluna e o de B máximo na linha".

Salienta-se que para a identificação dos Pontos de Equilíbrio observou-se os sinais (negativos ou positivos) dos Coeficientes Beta obtidos pelas Regressões Lineares.

Desse modo, procura-se evidenciar quais as estratégias (indicadores) que as empresas deveriam assumir para atingir um equilíbrio entre o desempenho econômico e social. Nash $(1950,1951)$ descreve que, tal como os jogos de soma zero, qualquer jogo bimatricial deve considerar a possibilidade de estratégias puras, quando há um ponto de equilíbrio de Nash, ou em estratégias mistas onde não á um ponto específico que a condição seja atendida. Considerando este determinação, admitiu-se os sistemas e será observado apenas a existência ou não de equilíbrio de Nash para estratégias puras.

Pautado sob essa afirmativa, formula-se as hipótese deste estudo, vinculada ao problema a ser investigado, sendo assim exposto: 
$\mathbf{H}_{0}$ : Não há um ponto de equilíbrio de Nash no jogo bimatricial de indicadores econômicos e indicadores sociais das empresas do setor de Utilidade Pública.

Se a hipótese nula $\mathrm{H}_{0}$, não se confirmar admitir-se-á hipótese $\mathrm{H}_{1}$ que é dada nos seguintes termos:

$\mathbf{H}_{1}$ : Há ao menos um ponto de equilíbrio de Nash no jogo bimatricial de indicadores econômicos e indicadores sociais das empresas do setor de Utilidade Pública.

Nesta concepção Simonsen (1994) corrobora para os momentos em que possa ocorrer a presença de mais um ponto de equilíbrio, podendo admitir estratégias mistas neste caso para se obter o melhor ponto de equilíbrio.

\section{METODOLOGIA}

Esta pesquisa apresenta por objetivo analisar a relação entre indicadores de desempenho econômico e social das empresas listadas no setor de Utilidade Pública da BM\&FBovespa mediante aplicação da teoria dos jogos para jogos bimatriciais.

Como população, delimitou-se todas as empresas pertencentes ao setor de Utilidade Pública, perfazendo um total de 75 empresas. Essa delimitação da população abarca o setor mais representativo de Janeiro de 2014, conforme a classificação setorial da BM\&FBovespa.

Portanto verifica-se que nem todas as empresas disponibilizavam as demonstrações contábeis consolidadas, bem como as informações necessárias para o cálculo dos indicadores de desempenho para os períodos em questão (2009 a 2011). Dessa forma, efetuou-se a exclusão destas empresas, sendo a amostra final composta por 26 empresas do setor de Utilidade Pública.

A utilização dos dados para a realização da análise consiste em um conjunto de indicadores de maior relevância para a mensuração do desempenho econômico e social das empresas.

Para caracterizar a situação econômica das empresas, utilizou-se os seguintes indicadores: Retorno do Ativo (ROA), Retorno do Patrimônio Líquido (ROE), Margem Líquida (ML), Lucro Por Ação (LPA) e Índice Preço/Lucro (P/L). A utilização destes indicadores está pautada em Brigham e Houston (1999), Schrickel (1999), Brigham, Gapenski e Ehrhardt (2001), Salazar e Benedicto (2004), Savytzky (2005), Brigham e Ehrhardt (2006), Assaf Neto (2009), Hoji (2012) e Silva (2012).

O desempenho social das empresas foi caracterizado sob a ótica da Demonstração do Valor Adicionado, devido à grande utilidade social desta demonstração. Os indicadores utilizados foram: Gastos com pessoal (GP), Gastos com tributos (GT), Quociente do Valor adicionado (QVA), Taxa de Variação do VA bruto (TVAB), Taxa de Variação Total do VA bruto (TVT) e o Potencial do Ativo em Gerar Riqueza (PAGR). Estes indicadores apresentam suporte teórico nos trabalhos de Bao e Bao (1998), Haller e Stolowy (1998), Santos (1999), Cosenza (2003), Aswegen, Steyn, Hamman (2005), Santos (2007), Nandi (2011).

Conforme elucidado acima, os resultados para a identificação do Ponto de Equilíbrio serão definidos mediante regressões lineares para determinar os valores dos Betas estandardizados, efetuadas por meio do software SPSS.

As regressões lineares serão efetuadas da seguinte forma: primeiramente realiza-se uma regressão entre um indicador social (variável dependente) com todos os indicadores econômicos (variáveis independentes). Dessa forma os coeficientes padronizados de Beta encontrados estarão dispostos nas linhas. Na sequência efetuam-se os mesmos procedimentos, por sua vez para os indicadores econômicos que serão considerados as variáveis dependentes e os indicadores sociais as variáveis independentes. Dessa forma, os coeficientes encontrados serão dispostos nas colunas. Neste contexto, para determinar o ponto de equilíbrio de Nash em um jogo bimatricial, Simonsen (1994: 414) salienta que se deve identificar "[...] onde o ganho de A seja máximo na coluna e o de B máximo na linha”. Dessa forma é identificado o Ponto de Equilíbrio do jogo, sendo para estes indicadores que as empresas devem posicionar as suas estratégias para alcançar um equilíbrio entre o desempenho econômico e social. 


\section{ANÁLISE DOS DADOS}

Busca-se nesta seção descrever a identificação dos Pontos de Equilíbrio de Nash de um jogo bimatricial entre indicadores econômico e indicadores sociais.

Para determinar o Ponto de Equilíbrio, definiu-se os valores com base na Regressão Linear Simples, considerando-se apenas os valores dos coeficientes padronizados Beta, pois esses indicam a relação existente entre as variáveis independentes e a variável dependente. $\mathrm{Na}$ Tabela 03, apresentam-se os resultados das regressões para o período de 2009 a 2011, assumindo como variáveis dependentes os indicadores econômicos e por variáveis independentes os indicadores sociais. Ou seja, primeira regressão com ROA na variável dependente e todos os indicadores sociais na variável independente, e assim sucessivamente.

Tabela 03 - Resultados das Regressões Lineares

\begin{tabular}{|c|c|c|c|c|c|c|c|c|c|c|}
\hline \multicolumn{11}{|c|}{2009} \\
\hline & \multicolumn{2}{|c|}{ ROA } & \multicolumn{2}{|c|}{ ROE } & \multicolumn{2}{|c|}{ ML } & \multicolumn{2}{|c|}{ LPA } & \multicolumn{2}{|l|}{ PL } \\
\hline & Beta Pad. & Sig. & Beta Pad. & Sig. & Beta Pad. & Sig. & Beta Pad. & Sig. & Beta Pad. & Sig. \\
\hline Constant & &, 246 & & ,766 & &, 295 & & ,967 & & ,000 \\
\hline GP &,- 201 & , 424 &, 050 & ,856 &,- 247 & ,379 &, 100 & ,699 & ,134 & , 195 \\
\hline GT &, 281 & ,381 &,- 198 &, 575 &, 313 & ,379 &,- 113 & ,731 & ,921 &, 000 \\
\hline QVA &,- 311 & , 168 &,- 135 & ,581 &,- 050 & ,837 &,- 207 & ,365 & 233 & ,016 \\
\hline TVAB &,- 041 & ,871 &, 013 & ,962 &,- 057 & ,838 &,- 094 & ,718 & ,203 & ,059 \\
\hline TVT &,- 001 & ,996 & ,077 & ,800 &,- 103 & ,735 &, 074 & ,795 & ,056 & ,617 \\
\hline PAGR &, 062 & ,843 & ,275 & ,433 &,- 217 & ,537 & ,394 & ,233 &,- 109 & ,396 \\
\hline $\mathbf{R}^{2}$ & &, 239 & &, 067 & &, 063 & & ,194 & &, 875 \\
\hline \multicolumn{11}{|c|}{2010} \\
\hline & \multicolumn{2}{|c|}{ ROA } & \multicolumn{2}{|c|}{ ROE } & \multicolumn{2}{|c|}{ ML } & \multicolumn{2}{|c|}{ LPA } & \multicolumn{2}{|l|}{ PL } \\
\hline & Beta Pad. & Sig. & Beta Pad. & Sig. & Beta Pad. & Sig. & Beta Pad. & Sig. & Beta Pad. & Sig. \\
\hline Constant & &, 190 & &, 687 & & ,319 & &, 165 & &, 000 \\
\hline GP &, 153 & ,593 &,- 306 & ,346 &,- 222 & ,495 & ,329 & ,276 &,- 383 &, 088 \\
\hline GT &,- 875 &, 035 & ,139 & ,753 &,- 083 & ,852 &,- 582 & ,164 &, 735 & ,021 \\
\hline QVA &,- 651 & ,019 &,- 249 & ,398 &,- 211 & ,475 &,- 223 & ,413 & ,275 &, 172 \\
\hline TVAB &, 015 & ,948 &,- 053 & ,842 & ,358 & , 186 &,- 315 & ,205 &,- 426 & ,024 \\
\hline TVT &,- 020 & ,935 & 277 & ,323 & ,207 & ,460 &,- 121 & ,637 &,- 479 & ,017 \\
\hline PAGR &, 726 & ,081 &,- 182 & ,687 &,- 199 & ,662 &, 790 & ,070 &,- 656 & ,041 \\
\hline $\mathbf{R}^{2}$ & & ,337 & &, 154 & & ,143 & & 275 & & ,618 \\
\hline \multicolumn{11}{|c|}{2011} \\
\hline & \multicolumn{2}{|c|}{ ROA } & \multicolumn{2}{|c|}{ ROE } & \multicolumn{2}{|l|}{ ML } & \multicolumn{2}{|c|}{ LPA } & \multicolumn{2}{|l|}{ PL } \\
\hline & Beta Pad. & Sig. & Beta Pad. & Sig. & Beta Pad. & Sig. & Beta Pad. & Sig. & Beta Pad. & Sig. \\
\hline Constant & & ,298 & & ,391 & & ,799 & &, 638 & & ,895 \\
\hline GP &,- 117 &, 784 &,- 067 & ,866 &,- 455 & ,326 &, 154 &, 736 &, 444 & ,312 \\
\hline GT &,- 379 & ,398 &,- 211 & ,616 &, 373 & ,441 &,- 364 & ,450 &,- 448 & ,330 \\
\hline QVA &,- 350 & ,226 &,- 537 &, 055 &, 075 & ,806 &,- 261 & ,397 &,- 138 & ,635 \\
\hline TVAB &,- 071 & ,793 &,- 108 & ,673 &,- 065 & ,823 &,- 062 & ,831 &, 034 & ,901 \\
\hline TVT &, 138 & ,664 &, 111 & ,709 &, 111 & ,746 &, 067 & ,843 &,- 142 & ,662 \\
\hline PAGR & ,065 & ,888 & , 169 & ,696 &,- 403 & ,421 & , 147 & ,766 &, 779 &, 109 \\
\hline $\mathbf{R}^{2}$ & & ,193 & & ,285 & & ,059 & & ,066 & &, 157 \\
\hline
\end{tabular}

Fonte: dados da pesquisa.

A princípio como se buscou analisar a possível existência de influência entre as variáveis independentes e a variável dependente, não foi levado em consideração o coeficiente de significância dos coeficientes Beta Padronizados. Para confirmação e prova de robustez deveria ser levado em consideração, entretanto este trabalho busca mais descrever o modelo de equilíbrio para fins gerenciais do que para fins de inferência estatística dos resultados.

$\mathrm{Na}$ Tabela 04, apresentam-se os resultados das regressões para o período de 2009 a 2011, assumindo como variáveis dependentes os indicadores sociais e por variáveis independentes os indicadores econômicos. Ou seja, primeira regressão com GP na variável 
dependente e todos os indicadores econômicos na variável independente, e assim sucessivamente.

Tabela 04 - Resultados das Regressões Lineares

\begin{tabular}{|c|c|c|c|c|c|c|c|c|c|c|c|c|}
\hline \multicolumn{13}{|c|}{2009} \\
\hline & \multicolumn{2}{|c|}{ GP } & \multicolumn{2}{|c|}{ GT } & \multicolumn{2}{|c|}{ QVA } & \multicolumn{2}{|c|}{ TVAB } & \multicolumn{2}{|c|}{ TVT } & \multicolumn{2}{|c|}{ PAGR } \\
\hline & $\begin{array}{l}\text { Beta } \\
\text { Pad }\end{array}$ & Sig. & $\begin{array}{l}\text { Beta } \\
\text { Pad }\end{array}$ & Sig. & $\begin{array}{l}\text { Beta } \\
\text { Pad }\end{array}$ & Sig. & $\begin{array}{l}\text { Beta } \\
\text { Pad }\end{array}$ & Sig. & $\begin{array}{l}\text { Beta } \\
\text { Pad }\end{array}$ & Sig. & $\begin{array}{l}\text { Beta } \\
\text { Pad }\end{array}$ & Sig. \\
\hline Const & & ,008 & & ,000 & & ,005 & & ,000 & & ,000 & &, 000 \\
\hline ROE &,- 348 & ,266 & , 105 & ,519 &,- 628 & 049 &,- 238 &, 472 & ,039 & ,902 & , 148 & ,620 \\
\hline ROA &, 108 & ,744 &,- 290 & 108 &, 446 &, 183 &, 354 &, 324 &, 028 & ,935 &,- 206 &, 520 \\
\hline ML &,- 035 & ,869 & ,041 & ,714 & ,059 & ,776 &,- 053 & ,814 &,- 098 & ,653 &,- 040 &, 845 \\
\hline LPA &, 055 & ,844 & 262 & ,084 &,- 270 & ,329 &,- 301 & ,315 & ,089 & ,755 &, 374 &, 171 \\
\hline PL & ,485 & ,034 & ,813 & ,000 & ,274 & ,208 & ,274 & ,244 & ,371 & ,107 & ,338 &, 116 \\
\hline $\mathbf{R}^{2}$ & \multicolumn{2}{|c|}{,221 } & \multicolumn{2}{|c|}{, 785} & \multicolumn{2}{|c|}{,244 } & \multicolumn{2}{|c|}{, 115} & \multicolumn{2}{|c|}{, 180} & \multicolumn{2}{|c|}{,282 } \\
\hline \multicolumn{13}{|c|}{2010} \\
\hline & \multicolumn{2}{|c|}{ GP } & \multicolumn{2}{|c|}{ GT } & \multicolumn{2}{|c|}{ QVA } & \multicolumn{2}{|c|}{ TVAB } & \multicolumn{2}{|c|}{ TVT } & \multicolumn{2}{|c|}{ PAGR } \\
\hline & $\begin{array}{l}\text { Beta } \\
\text { Pad }\end{array}$ & Sig. & $\begin{array}{l}\text { Beta } \\
\text { Pad }\end{array}$ & Sig. & $\begin{array}{l}\text { Beta } \\
\text { Pad }\end{array}$ & Sig. & $\begin{array}{l}\text { Beta } \\
\text { Pad }\end{array}$ & Sig. & $\begin{array}{l}\text { Beta } \\
\text { Pad }\end{array}$ & Sig. & $\begin{array}{l}\text { Beta } \\
\text { Pad }\end{array}$ & Sig. \\
\hline Const & & ,005 & & ,000 & & ,005 & & ,000 & & ,000 & &, 000 \\
\hline $\mathrm{ROA}$ &,- 204 & 609 &,- 434 & ,273 &,- 156 & 669 & ,298 & ,319 & ,000 &, 119 &,- 328 & ,382 \\
\hline ROE &,- 089 & ,791 &, 403 & 228 &,- 463 &, 142 &,- 588 &, 026 & 119 &, 185 &, 379 & ,234 \\
\hline $\mathrm{ML}$ &,- 093 & ,694 &, 151 & ,513 &,- 029 & ,894 &, 179 & ,310 & ,185 & ,319 &, 126 &, 568 \\
\hline LPA &, 103 & ,826 & ,430 &, 355 &,- 286 & ,509 &,- 903 &, 016 & ,319 &, 174 &, 517 & ,246 \\
\hline PL &,- 202 & ,368 &,- 155 & ,478 &,- 183 & ,373 &,- 453 & 011 &, 174 &, 001 &,- 327 &, 125 \\
\hline $\mathbf{R}^{2}$ & \multicolumn{2}{|c|}{, 082} & \multicolumn{2}{|c|}{, 120} & \multicolumn{2}{|c|}{,231 } & \multicolumn{2}{|c|}{,496 } & \multicolumn{2}{|c|}{,492 } & \multicolumn{2}{|c|}{,203 } \\
\hline \multicolumn{13}{|c|}{2011} \\
\hline & \multicolumn{2}{|c|}{ GP } & & & & & & & & & & \\
\hline & $\begin{array}{l}\text { Beta } \\
\text { Pad }\end{array}$ & Sig. & $\begin{array}{c}\text { Beta } \\
\text { Pad }\end{array}$ & Sig. & $\begin{array}{c}\text { Beta } \\
\text { Pad }\end{array}$ & Sig. & $\begin{array}{c}\text { Beta } \\
\text { Pad }\end{array}$ & Sig. & $\begin{array}{c}\text { Beta } \\
\text { Pad }\end{array}$ & Sig. & $\begin{array}{l}\text { Beta } \\
\text { Pad } \\
\end{array}$ & Sig. \\
\hline Const & & ,005 & & ,000 & & ,000 & &, 000 & & ,000 & &, 000 \\
\hline $\mathrm{ROE}$ &,- 585 & ,203 &,- 785 & ,089 & ,566 & ,166 & ,216 & ,653 &,- 538 & ,243 &,- 147 &, 745 \\
\hline ROA &, 112 & ,728 & ,480 &, 140 &,- 786 & ,011 &,- 159 & ,643 &, 515 &, 122 & 249 &, 440 \\
\hline $\mathrm{ML}$ &,- 015 & ,945 &, 154 & ,485 &,- 111 &, 574 &,- 085 &, 718 &, 128 &, 569 &, 055 & ,803 \\
\hline LPA &, 386 & ,356 & ,527 & ,206 &,- 672 & ,078 &,- 201 & ,649 &, 516 & ,223 &, 102 &, 805 \\
\hline PL &,- 144 & ,503 & ,046 & ,828 & ,005 & ,980 &,- 050 &, 825 & 079 & ,713 & ,334 & 130 \\
\hline$\overline{\mathbf{R}^{2}}$ & & & & & & & & & & & & \\
\hline
\end{tabular}

Fonte: dados da pesquisa.

Realizadas as Regressões Lineares, na sequência, determina-se o ganho máximo disposto na coluna para o Jogador A (indicadores econômicos) e o ganho máximo de disposto na linha para o Jogador B (indicadores sociais) conforme elencado por Simonsen (1994). Dessa maneira encontra-se o Ponto de Equilíbrio de Nash para um jogo bimatricial, de modo que as empresas posicionem suas estratégias para tais indicadores afim de alcançar um equilíbrio entre o desempenho econômico e o desempenho social.

Apresentam-se na Tabela 05 os resultados para o Ponto de Equilíbrio de Nash para o período de 2009 a 2011. 
Tabela 05 - Ponto de Equilíbrio de Nash do setor de Utilidade Pública

\begin{tabular}{|c|c|c|c|c|c|c|}
\hline \multicolumn{7}{|c|}{2009} \\
\hline & ROA & ROE & ML & LPA & $\mathrm{P} / \mathrm{L}$ & $\operatorname{Max} b_{i j}$ \\
\hline GP & $(-0,348 ;-0,201)$ & $(0,108 ; 0,050)$ & $(-0,035 ;-0,247)$ & $(0,055 ; 0,100)$ & $(0,485 ; \mathbf{0 , 1 3 4})$ & 0,134 \\
\hline GT & $(0,105 ; 0,281)$ & $(-0,290 ;-0,198)$ & $(0,041 ; 0,313)$ & $(0,262 ;-0,113)$ & $(0,813 ; 0,921)$ & 0,921 \\
\hline QVA & $(-0,628 ;-0,311)$ & $(\mathbf{0 , 4 4 6} ;-0,135)$ & $(\mathbf{0 , 0 5 9} ;-0,050)$ & $(-0,270 ;-0,207)$ & $(0,274 ; \mathbf{0 , 2 3 3})$ & 0,233 \\
\hline TVAB & $(-0,238 ;-0,041)$ & $(0,354 ; 0,013)$ & $(-0,053 ;-0,057)$ & $(-0,301 ;-0,094)$ & $(0,274 ; \mathbf{0 , 2 0 3})$ & 0,203 \\
\hline TVT & $(0,039 ;-0,001)$ & $(0,028 ; \mathbf{0 , 0 7 7})$ & $(-0,098 ;-0,103)$ & $(0,089 ; 0,074)$ & $(0,371 ; 0,056)$ & $\mathbf{0 , 0 7 7}$ \\
\hline PAGR & $(\mathbf{0 , 1 4 8} ; 0,062)$ & $(-0,206 ; 0,275)$ & $(-0,040 ;-0,217)$ & $(0,374 ; 0,394)$ & $(0,338 ;-0,109)$ & 0,394 \\
\hline $\operatorname{Max} a_{i j}$ & 0,148 & 0,446 & 0,059 & 0,374 & $\mathbf{0 , 8 1 3}$ & \\
\hline \multicolumn{7}{|c|}{2010} \\
\hline & ROA & ROE & ML & LPA & $\mathrm{P} / \mathrm{L}$ & $\operatorname{Max} b_{i j}$ \\
\hline GP & $(-0,204 ; 0,153)$ & $(-0,089 ;-0,306)$ & $(-0,093 ;-0,222)$ & $(0,103 ; \mathbf{0 , 3 2 9})$ & $(-0,202 ;-0,383)$ & 0,329 \\
\hline GT & $(-0,434 ;-0,875)$ & $(\mathbf{0 , 4 0 3} ; 0,139)$ & $(0,151 ;-0,083)$ & $(0,430 ;-0,582)$ & $(-0,155 ; 0,735)$ & $\mathbf{0 , 7 3 5}$ \\
\hline QVA & $(-0,156 ;-0,651)$ & $(-0,463 ;-0,249)$ & $(-0,029 ;-0,211)$ & $(-0,286 ;-0,223)$ & $(-0,183 ; \mathbf{0 , 2 7 5})$ & 0,275 \\
\hline TVAB & $(\mathbf{0 , 2 9 8} ; 0,015)$ & $(-0,588 ;-0,053)$ & $(0,179 ; 0,358)$ & $(-0,903 ;-0,315)$ & $(-0,453 ;-0,426)$ & $\mathbf{0 , 3 5 8}$ \\
\hline TVT & $(-0,476 ;-0,020)$ & $(0,338 ; \mathbf{0 , 2 7 7})$ & $(0,177 ; 0,207)$ & $(0,486 ;-0,121)$ & $(-0,633 ;-0,479)$ & 0,277 \\
\hline PAGR & $(-0,328 ; 0,726)$ & $(0,379 ;-0,182)$ & $(0,126 ;-0,199)$ & $(0,517 ; 0,790)$ & $(-0,327 ;-0,656)$ & 0,790 \\
\hline $\operatorname{Max} a_{i j}$ & 0,298 & $\mathbf{0 , 4 0 3}$ & 0,179 & $\mathbf{0 , 5 1 7}$ & $-0,155$ & \\
\hline \multicolumn{7}{|c|}{2011} \\
\hline & ROA & $\mathrm{ROE}$ & ML & LPA & $\mathrm{P} / \mathrm{L}$ & $\operatorname{Max} b_{i j}$ \\
\hline GP & $(-0,585 ;-0,117)$ & $(0,112 ;-0,067)$ & $(-0,015 ;-0,455)$ & $(0,386 ; 0,154)$ & $(-0,144 ; \mathbf{0 , 4 4 4 )}$ & 0,444 \\
\hline GT & $(-0,785 ;-0,379)$ & $(0,480 ;-0,211)$ & $(0,154 ; 0,373)$ & $(\mathbf{0 , 5 2 7} ;-0,364)$ & $(0,046 ;-0,448)$ & $\mathbf{0 , 3 7 3}$ \\
\hline QVA & $(\mathbf{0 , 5 6 6} ;-0,350)$ & $(-0,786 ;-0,537)$ & $(-0,111 ; \mathbf{0 , 0 7 5})$ & $(-0,672 ;-0,261)$ & $(0,005 ;-0,138)$ & 0,075 \\
\hline TVAB & $(0,216 ;-0,071)$ & $(-0,159 ;-0,108)$ & $(-0,085 ;-0,065)$ & $(-0,201 ;-0,062)$ & $(-0,050 ; \mathbf{0 , 0 3 4 )}$ & 0,034 \\
\hline TVT & $(-0,538 ; \mathbf{0 , 1 3 8})$ & $(\mathbf{0 , 5 1 5} ; 0,111)$ & $(0,128 ; 0,111)$ & $(0,516 ; 0,067)$ & $(0,079 ;-0,142)$ & 0,138 \\
\hline PAGR & $(-0,147 ; 0,065)$ & $(0,249 ; 0,169)$ & $(0,055 ;-0,403)$ & $(0,102 ; 0,147)$ & $(0,334 ; 0,779)$ & 0,779 \\
\hline $\operatorname{Max} a_{i j}$ & 0,566 & 0,515 & 0,154 & 0,527 & 0,334 & \\
\hline
\end{tabular}

Fonte: Dados da pesquisa.

Conforme os dados apresentados na Tabela 05, a primeira combinação encontrada que induz a ganhos no desempenho econômico e no desempenho social de maneira conjunta é adotar como estratégia a combinação do indicador de Lucro por Ação com o Potencial do Ativo em Gerar Riqueza. Outra estratégia que gera ganhos comuns a indicadores econômicos e sociais adotada pelas empresas em 2009 é dada pela combinação do Índice Preço/Lucro com os Gastos com Tributos.

No período de 2010, identificaram-se três estratégias que acarretaram em um equilíbrio para as duas formas de avaliação de desempenho analisadas na pesquisa. Infere-se que as empresas adotaram por estratégias a combinação da Margem Líquida com a Taxa de Variação do Valor Adicionado Bruto, o Lucro por Ação com o Potencial do Ativo em Gerar Riqueza e ainda o Índice Preço/Lucro em combinação com os Gastos com Tributos.

Para o período de 2011 as empresas do setor de Utilidade Pública adotaram duas combinações estratégicas que combinadas geraram ganhos para ambos os grupos de indicadores. Estas combinações foram: Margem Líquida com os Gastos com Tributos e ainda o Índice Preço/Lucro com o Potencial do Ativo em Gerar Riqueza.

Constata-se que as empresas do setor de Utilidade Pública que utilizam por foco o indicador do Quociente do Valor Adicionado e da Taxa de Variação Total do Valor Adicionado, tendem a prejudicar todos os seus indicadores econômicos. Assim como, o Retorno sobre o Ativo e o Retorno sobre o Patrimônio Líquido tendem a prejudicar os indicadores sociais. Esta constatação é efetuada, pois, estes indicadores não se encontram em nenhuma das estratégias adotadas pelas empresas e identificadas no Ponto de Equilíbrio de Nash.

Estes são os Pontos de Equilíbrio de Nash identificados nos jogos, para os quais as empresas podem posicionar as suas estratégias, de modo a alcançar um equilíbrio entre o desempenho econômico e o desempenho social. Onde não se obtêm ganhos para satisfazer 
tanto o primeiro conjunto de indicador e o segundo conjunto de indicadores sem onerar nenhuma das duas categorias de informações.

Conforme os dados apresentados, é possível perceber que para todos os períodos analisados encontrou-se no mínimo um Ponto de Equilíbrio. De modo que são para estes indicadores que as empresas podem posicionar as suas estratégias, para alcançar um equilíbrio entre o desempenho econômico e social, sem causar desconforto a nenhum dos jogadores.

Este achado implica na rejeição da hipótese nula $\left(\mathrm{H}_{0 \mathrm{~b}}\right)$ e aceitação da hipótese $\left(\mathrm{H}_{1 \mathrm{~b}}\right)$ assumindo-se que há pelo menos um ponto de equilíbrio de Nash no jogo bimatricial de indicadores econômicos e indicadores sociais. Corroborando dessa forma com a premissa estabelecida por Nash $(1950,1951)$ quando definiu que qualquer jogo pautado em estratégias mistas apresenta um Ponto de Equilíbrio. Simonsen (1994) alerta para os momentos em que possa ocorre a identificação de mais de um Ponto de Equilíbrio no jogo. Dessa maneira a combinação de estratégias dos indicadores econômicos com os indicadores sociais acarreta um equilíbrio do desempenho econômico e desempenho social das empresas.

\section{CONCLUSÃO}

Durante longos períodos de tempo a grande preocupação das empresas versava sobre o seu desempenho econômico e financeiro, por sua vez, surge uma nova perspectiva enfatizando que não é mais o lucro o fator mais importante para a análise de desempenho. Desse modo insere-se a discussão em torno da sustentabilidade empresarial, que está pautada em um equilíbrio econômico, social e ambiental para as empresas.

Perante esta discussão a presente pesquisa apresenta por objetivo identificar o Ponto de Equilíbrio de Nash no jogo bimatricial de indicadores econômicos e indicadores sociais das empresas do setor de Utilidade Pública listadas na BM\&FBovespa. Realizou-se a pesquisa com uma amostra de 26 empresas. A escolha dos indicadores para a avaliação de desempenho econômico está pautada em indicadores de rentabilidade e de mercado e para a avaliação de desempenho social, utilizou-se os indicadores oriundos da DVA.

O modelo descrito trouxe como grande dificuldade a estimação dos valores para as matrizes de jogos. Estimar os ganhos entre os indicadores foi a grande dificuldade encontrada no trabalho. Para tanto se utilizou para estimação dos parâmetros da matriz de jogo os parâmetros padronizados da regressão linear múltipla. A utilização dos coeficientes padronizados leva em consideração que os coeficientes estimados de uma regressão são interferidos diretamente por meio da magnitude ou grandeza da variável. Por outro lado os coeficientes padronizados estão livres desta implicação. Possibilita assim a comparação e, portanto sua utilização na estimação dos pesos da matriz de jogos.

Os resultados obtidos destacaram a existência de pelo menos um ponto de equilíbrio para os anos analisados, e em especial os modelos propuseram dois pontos de equilíbrio para 2009 e 2011, e três pontos para 2010. Este número maior de números favorece em um possível processo de tomada de decisão por parte dos usuários desta informação.

Destaca-se que o modelo proposto confirmou a hipótese da existência de pelo menos um ponto de equilíbrio, e assim a pesquisa consegue satisfazer seu objetivo de que é possível atender tanto aos aspectos econômicos quanto aos aspectos sociais das empresas quando se toma como informações básicas os indicadores. Questionamentos que podem ser tratados com maior profundidade é referente a importância do coeficiente de significância das regressões para o modelo, além de levar em consideração algum critério de obtenção do ponto de equilíbrio de Nash para estratégias mistas.

\section{REFERENCIAS}

[1] Accounting Standards Steering Committee. (1975). The corporate report. London.

[2] Arlow, P. \& Gannon, M. J. (1982). Social responsiveness, corporate stru ture and economic performance. Academy of Management Review, 7 (2): 235-241. 
[3] Assaf Neto, A. 2009. Finanças corporativas e valor. 4. ed. São Paulo: Atlas.

[4] Aswegen, N. V.; Steyn, B. W. \& Hamman, W. D. (2005). Trends in the distribution of added value of listed industrial companies - 1990 to 2002. South African Journal of Business Management, 36 (2): 85-94.

[5] Aupperle, K. E.; Carroll, A. B. \& Hatfield, J. D. (1985). An empirical examination of the relationship between corporate social responsibility and profitability. Academy of Management Journal, 28 (2): 446-463.

[6] Bao, B.-H.\& Bao, D.-H. (1998). Usefulness of value added and abnormal economic earnings: an empirical examination. Journal of Business Finance \& Accounting, 25 (12): 251-264.

[7] Baumgartner, R. J. \& Ebner, D. (2010). Corporate Sustainability strategies: sustainability profiles and maturity levels. Sustainable Development, 18: 76-89.

[8] Bontempo, M. T. (1997). Teoria dos jogos aplicada a contabilidade. Caderno de Estudos [online], 9 (15): 35-40.

[9] Brandt,F.; Fischer,F.; Harrenstein,P. \&Shoham,Y.(2009). Ranking games. Artificial Intellingence, 173: 221-239.

[10] Brigham, E. F. \&Ehrhardt, M. C. (2006). Administração Financeira: teoria e Prática. São Paulo: Pioneira Thomson Learning.

[11] Brigham, E.F.; Gapenski, L. \& Ehrhardt,M. (2001). Administração Financeira: teoria e Prática. São Paulo: Atlas.

[12] Brigham, E.\& Houston, J. (1999). Fundamentos da moderna administração financeira. Rio de Janeiro: Campus.

[13] Callado, A. L. C. (2010). Modelo de mensuração de sustentabilidade empresarial: uma aplicação em vinícolas localizadas na Serra Gaúcha. 2010. 216 f. Tese (Doutorado em Agronegócios). Porto Alegre: Universidade Federal do Rio Grande do Sul.

[14] Callado, A. L. C. \& Feinsterseifer, J. E. (2010). Mensuração de sustentabilidade através do Grid de Sustentabilidade Empresarial (GSE): um estudo no setor vinícula. In: Sociedade Brasileira de Economia, Administração e Sociologia Rural, 48., 2010, Anais... Campo Grande: SOBER.

[15] Carroll, A. B. (1991). The pyramid of corporate social responsibility: toward the moral management of organizational stakeholders. Business Horizons.

[16] Clarke-Sather, A. R.; Hutchins, M. J.; Zhang, Q., Gershenson, J. K \&Sutherland, J. W. (2011). Development of social, environmental, and economic indicators for a small/médium enterprise. International Journal of Accounting and Informations Management, 19 (3): 247-266.

[17] Cosenza, J. P. (2003). A eficácia informativa da demonstração do valor adicionado. Revista Contabilidade \& Finanças, Edição comemorativa: 7-29.

[18] Dyllick, T. \& Hockerts, K. (2002). Beyond the business case for corporate sustainability. Business Strategy and the environment, 11: 130-141.

[19] Fiani, R. (2004). Teoria dos jogos: para cursos de Administração e economia. Rio de Janeiro, Elsevier.

[20] Haller, A. \& Stolowy, H. (1998). Value added in financial accounting a comparative study between Germany and France: 1-15.

[21] Hoji, M. (2012). Administração Financeira e Orçamentária: matemática financeira aplicada, estratégias financeiras, orçamento empresarial. 10. ed. São Paulo: Atlas.

[22] Ioannou, I. \& Serafeim, G. (2012). The consequences of mandatory corporate sustainability reporting.

[23] Krajnc, D.\& Glavic, P. (2005). A model for integrated assessment of sustainable development. Resources, Conservation and Recycling, 43 (2): 189-208.

[24] Lee, K.-H. \&Saen, R. F. (2012). Measuring corporate sustainability management: a data envelopment analysis approach. Int. J. Production Economics, 140: 219-226. 
[25] Lins, M. P. E. \& Calôba, G. M. (2006). Programação linear: com aplicações em teoria dos jogos e avaliação de desempenho (data envelopment analysis). Rio de Janeiro: Interciência.

[26] Lo, Shih-Fang. 2010. Performance evaluation for sustainable businnes a profitability and marketanbility framework. Corporate social resposibility and environmental management, 17: 311-319.

[27] MAROCO, J. (2003). Análise estatística: com utilização do SPSS. 2. ed. Lisboa: Edições Sílabo.

[28] Nandi, K. C. (2011). Performance measures: an application of value added statement. The IUP Journal of Operations Management, 10 (3): 39-62.

[29] Nash, J. (1950). Non-cooperative games. Dissertation (Doctor of Philosophy) Faculty of Princeton.

[30] Nash, J. (1951). Non-cooperative games. The annals of mathematics, 54 (2): 286-295.

[31] Preston, L E. \& O'Bannon, D. P. (1997). The corporate social-financial performance relationship. Business and society, 36 (4): 419-429.

[32] Salazar, J. N. A. \& Benedicto, G. C. (2004). Contabilidade Financeira. São Paulo: Pioneira Thompson Learning.

[33] Santos, A. (2007). Demonstração do Valor Adicionado: como elaborar e analisar a DVA. São Paulo: Atlas.

[34] Savytzky, T. (2005). Análise de Balanços: Método prático. 3. ed. Curitiba: Juruá Editora.

[35] Schrickel, W.K. (1999). Demonstrações Financeiras: abrindo a caixa-preta.São Paulo: Atlas.

[36] Silva, A. A. (2012). Estrutura, análise e interpretação das demonstrações contábeis. 3. ed. São Paulo: Atlas.

[37] Simonsen, M. H. (1994). Ensaios analíticos. Rio de Janeiro: Editora da Fundação Getulio Vargas.

[38] Ullmann, A. A. (1985). Data in search of a heory: a critical examination of the relationships among social performance, social disclosure, and economic performance of U.S. firms. Academy of management review, 10 (3): 540-557.

[39] Von Neumann, J.\& Morgenstern, O. (1953). Theory of games and economic behavior. 3. ed. Princeton: Princeton University Press. 